\title{
Age- and Sex-Specific Impact of Health Literacy on Healthy Lifestyle Characteristics among Japanese Residents in a Rural Community
}

\author{
Hirohide Yokokawa1 ${ }^{*}$, Motoyuki Yuasa ${ }^{2}$, Hironobu Sanada ${ }^{3,4}$, Teruhiko Hisaoka1, \\ Hiroshi Fukuda 1 \\ ${ }^{1}$ Department of General Medicine, Juntendo University School of Medicine, Tokyo, Japan \\ ${ }^{2}$ Department of Public Health, Juntendo University School of Medicine, Tokyo, Japan \\ ${ }^{3}$ Division of Health Science Research, Fukushima Welfare Federation of Agricultural Cooperatives, Fukushima, \\ Japan \\ ${ }^{4}$ Department of Tumor and Host Bioscience, Fukushima Medical University School of Medicine, Fukushima, \\ Japan \\ Email: ${ }^{*}$ yokokawa@pa3.so-net.ne.jp
}

Received 24 April 2015; accepted 9 June 2015; published 12 June 2015

Copyright (C) 2015 by authors and Scientific Research Publishing Inc.

This work is licensed under the Creative Commons Attribution International License (CC BY).

http://creativecommons.org/licenses/by/4.0/

(c) (i) Open Access

\section{Abstract}

The study aimed to examine age- and sex-specific associations between health literacy (HL) and healthy lifestyle characteristics. The cross-sectional study was conducted to examine a total 1348 Japanese participants (613 male and 735 female) who took the health check-up program in April 2013, at Tsumagoi Village, Japan. Information was collected by self-administered questionnaire on three communicative and two critical HL items, and healthy lifestyle characteristics listed in Breslow's seven health practices. In multivariate logistic analysis, high HL $(\geq 18)$ was significantly associated with healthy lifestyle characteristics in both non-elderly ( $<65$ years old) and elderly ( $\geq 65$ years old) [Odds ratio $(O R)=1.40,95 \%$ Confidence interval $(\mathrm{CI})=1.13-1.75$ for non-elderly, $\mathrm{OR}=1.34,95 \% \mathrm{CI}=1.02-1.76$ for elderly $]$, as well as female participants $[\mathrm{OR}=1.43,95 \% \mathrm{CI}=$ 1.06 - 1.94), while the significance was borderline in male participants $(O R=1.34,95 \% \mathrm{CI}=0.96$ 1.88). Among $5 \mathrm{HL}$ items, the ability to extract health-related information was significantly associated with healthy lifestyle characteristics $(O R=1.52,95 \% \mathrm{CI}=1.11-2.07$ for non-elderly, $\mathrm{OR}=$ $1.55,95 \% \mathrm{CI}=1.04-2.30$ for elderly, $\mathrm{OR}=1.95,95 \% \mathrm{CI}=1.28-2.97$ for female participants. HDL-C concentration in the high HL group was significantly higher than that in the low HL group. Among healthy lifestyle characteristics, proportions of smoking behavior (non-smoker), exercise fre-

*Corresponding author.

How to cite this paper: Yokokawa, H., Yuasa, M., Sanada, H., Hisaoka, T. and Fukuda, H. (2015) Age- and Sex-Specific Impact of Health Literacy on Healthy Lifestyle Characteristics among Japanese Residents in a Rural Community. Health, 7, 679-688. http://dx.doi.org/10.4236/health.2015.76081 
quency (twice or more per week), and sleep duration (7 - 8 hours) were significantly higher in the high HL group than in the low HL group. The present study revealed a positive association between HL and healthy lifestyle characteristics, which suggests that people are likely to engage in health-promoting behaviours based on access and ability to understand medical information for both age groups and female participants. Significant findings among female participants may be emphasized since it's important from the entire family's perspective as women are the caretakers of the family. These findings may indicate the importance of comprehensive assessment, including HL, for health promotion in the community.

\title{
Keywords
}

\author{
Epidemiology, Health Promotion, Health Literacy, Community Health, Lifestyle
}

\section{Introduction}

In recent decades, developed as well as developing countries have faced a dramatic increase in the number of adults suffering from non-communicable diseases (NCDs) such as diabetes, cardiovascular disease (CVD), and chronic kidney disease (CKD) [1]. Although the growing human and economic costs of NCDs have been recognized, and action plans to prevent NCDs have been outlined, the outcomes have been limited. Unhealthy lifestyles are likely to contribute to the development of NCDs, and the importance of lifestyle modification has been emphasized through advanced communication tools [2].

Health information is important for people to understand and engage in the management of their own health status. With increasing health information available through media reports and the Internet, many health information resources are easily distributed to the general population [3]-[5]. However, adequate use of these resources depends on an individual's skill in finding and applying information about their health issues [6]. These skills have been conceptualized as health literacy (HL). Based on the World Health Organization (WHO) definition, a model of HL has been proposed that includes three levels, and assumes both individual and population benefits at each level: functional, communicative, and critical literacy [7]. Individuals with adequate $\mathrm{HL}$ are considered likely to adopt healthy lifestyles [8], and a brief questionnaire was developed to easily assess HL [9]. However, only a few studies have estimated the association between HL and healthy lifestyle characteristics among Japanese people and reports that broadly include women and the elderly in rural communities are especially limited [9] [10].

This study aimed to examine age- and sex-specific associations between HL and healthy lifestyle characteristics among a Japanese community-dwelling population using a concise, newly developed questionnaire.

\section{Subjects and Methods}

\subsection{Participants}

This is a study using the baseline data of a prospective cohort study carried out in Tsumagoi Village, Gunma Prefecture, Japan, from April 2013 to April 2014. In the study, baseline survey data (April 2013) was analyzed. Tsumagoi Village is located in the northern region of Gunma Prefecture, Japan, and had a population of about ten thousand in 2013. The proportion of the elderly population (in the total population) in 2013 was $25.1 \%$.

Participants were recruited from the community-dwelling population living in Tsumagoi Village. This study was conducted as part of a local government-sponsored, multi-phasic health checkup program aimed at the general population based on the Law of Health and Medical Services for the Elderly. Of the 1450 individuals aged $>30$ years who took part in the program during the baseline survey period (April 2013), 1348 agreed to enroll in the study.

\subsection{Variables}

Body height and weight were measured in the standing position. Body mass index (BMI) was calculated based on body weight $(\mathrm{kg})$ divided by height squared $\left(\mathrm{m}^{2}\right)$. Both systolic (SBP) and diastolic blood pressure (DBP) 
were obtained as the mean of two measurements on the upper arm after the participant had been seated for at least five minutes. We also collected information for the following lipid-related items: total cholesterol (mg/dL; TC), high-density lipoprotein-cholesterol (mg/dL; HDL-C), and triglycerides (mg/dL; TG). Low-density lipoprotein-cholesterol (mg/dL; LDL-C) was estimated using the Friedwald equation ([TC] - [HDL-C] - [TG/5]) [11]. Glycosylated hemoglobin A1c (Japanese Diabetes Society [JDS; \%]) levels were determined by high-performance liquid chromatography using an automated analyzer. HbA1c (JDS \%) was converted to a National Glycohemoglobin Standard Program (NGSP) equivalent value using the following formula: HbA1c (NGSP \%) $=1.02 \times \operatorname{HbA} 1 \mathrm{c}(\mathrm{JDS} \%)+0.25 \%[12]$.

We interviewed participants regarding their medical histories for atherosclerotic complications (cardiovascular or cerebrovascular diseases) and lifestyle related disorders (hypertension, dyslipidemia, diabetes mellitus). We then asked participants to complete self-administrated questionnaires, which included HL and healthy lifestyle characteristics listed in Breslow's seven health practices, such as alcohol consumption, smoking behavior, exercise frequency, obesity (BMI), sleep duration, breakfast, and snacks between meals [13].

To evaluate HL, a validated questionnaire that included three items for communicative HL (items i - iii) and two items for critical HL (items iv - v) was used [9]. In the previous report related with the questionnaire, Cronbach's $\alpha$ of communicative and critical HL scales were 0.77 and 0.65 [9]. Participants may fill the questionnaire easily because the questionnaire is concisely composed with these 5 items. These items asked whether the participant would be able to i) collect health-related information from various sources, ii) extract the relevant information, iii) understand and communicate the obtained information, iv) consider the credibility of the information, and v) make decisions based on the information, specifically in the context of health-related issues. The participant rated each item on a 5-point Likert scale ranging from 1 (strongly disagree) to 5 (strongly agree).

We defined healthy lifestyle characteristics as the following responses based on Breslow's seven health practices [13] [14]: alcohol consumption (less than one drink per day), smoking behavior (non-smoker), exercise frequency (twice or more per week), BMI (18.5 - 24.9), sleep duration (7 - 8 hours), breakfast (every morning), and snacks between meals (no).

\subsection{Statistical Analysis}

Variables are presented as mean \pm standard deviation (SD) for continuous variables or prevalence (\%) for categorical variables. Total HL score was dichotomized based on median score (low HL: $<18$, high HL: $\geq 18$ ). For age-specific analysis, we defined "elderly" as being $\geq 65$ years old. We used the t-test for continuous variables and the chi-square test or Fisher's exact test for comparisons of proportions between two groups The Dunnett method were used to analyze the correlation between total HL score and total number of healthy characteristics. To estimate the potential of HL to promote a healthy lifestyle with 6 - 7 healthy characteristics, which seems the most likely to be present in even the healthiest lifestyle [14], logistic regression analysis was performed using the following models in both age and sex categories: Model 1 was adjusted for sex in age-specific analysis or age (years old) in sex-specific analysis, total HL score ( $\geq 18$ vs. $<18$ ), atherosclerotic complications (cardiovascular and cerebrovascular diseases), and medical history of lifestyle-related disorders (antihypertensive drug use, antidiabetic drug use, antidyslipidemic drug use). Model 2 was adjusted for sex in age-specific analysis or age (years old) in sex-specific analysis, five HL item scores, atherosclerotic complications (cardiovascular and cerebrovascular diseases), and medical history of lifestyle-related disorders (antihypertensive drug use, antidyslipidemic drug use, antidiabetic drug use).

All statistical analyses were performed using the Statistical Package for Social Sciences version 22 (IBM SPSS Inc., Chicago, IL, USA). $P<0.05$ was considered statistically significant.

\subsection{Ethics}

This survey was conducted according to the Ethical Guidelines for Epidemiological Studies established by the Japanese government [15], and the Ethics Committee of Juntendo University approved the research protocol (No. 833). We obtained informed consent from all participants.

\section{Results}

Table 1 shows basic characteristics of participants stratified by HL levels. Mean age and male proportion were 
Table 1. Patient characteristics stratified by health literacy level $(\mathrm{N}=1348)$.

\begin{tabular}{|c|c|c|c|c|c|}
\hline \multirow[b]{3}{*}{ Age (years) } & \multicolumn{4}{|c|}{ Mean $( \pm \mathrm{SD})$ or $\mathrm{N}(\%)$} & \multirow{3}{*}{$P^{\mathrm{a}}$} \\
\hline & \multicolumn{2}{|c|}{$\begin{array}{l}\text { Low health literacy }(<18) \\
\qquad(\mathrm{N}=623)\end{array}$} & \multicolumn{2}{|c|}{$\begin{array}{l}\text { High health literacy }(\geq 18) \\
\qquad(\mathrm{N}=725)\end{array}$} & \\
\hline & 66.4 & $(15.1)$ & 66.5 & $(14.7)$ & \\
\hline Elderly participants ( $\geq 65$ years old) & 406 & $(65.2)$ & 480 & $(66.2)$ & \\
\hline Sex (male) & 299 & $(48.0)$ & 314 & $(43.3)$ & \\
\hline \multicolumn{6}{|l|}{ Anthropometric measurements } \\
\hline Body mass index (BMI) & 23.9 & (3.6) & 23.5 & (3.3) & \\
\hline \multicolumn{6}{|l|}{ Atherosclerotic complications } \\
\hline Cardiovascular disease & 27 & (4.3) & 22 & $(3.0)$ & \\
\hline Cerebrovascular disease & 19 & $(3.0)$ & 19 & $(2.6)$ & \\
\hline \multicolumn{6}{|l|}{ Hypertension-related factors } \\
\hline Systolic blood pressure (mmHg) & 136.1 & $(17.6)$ & 136.3 & $(17.8)$ & \\
\hline Diastolic blood pressure (mmHg) & 75.4 & $(10.1)$ & 76.0 & $(11.2)$ & \\
\hline Antihypertensive drug use (yes) & 265 & $(42.5)$ & 287 & (39.6) & \\
\hline \multicolumn{6}{|l|}{ Lipid-related items } \\
\hline High-density lipoprotein cholesterol (mg/dL) & 59.7 & $(15.2)$ & 61.5 & $(16.2)$ & * \\
\hline Low-density lipoprotein cholesterol (mg/dL) & 123.7 & $(30.4)$ & 121.6 & $(29.5)$ & \\
\hline Triglycerides (mg/dL) & 139.8 & $(94.2)$ & 136.6 & $(83.1)$ & \\
\hline Antidyslipidemic drug use (yes) & 96 & $(15.4)$ & 115 & $(15.9)$ & \\
\hline \multicolumn{6}{|l|}{ Diabetes-related items } \\
\hline Hemoglobin A1c (\%) & 5.61 & $(0.57)$ & 5.60 & $(0.59)$ & \\
\hline Antidiabetic drug use (yes) & 18 & $(2.9)$ & 28 & (3.9) & \\
\hline \multicolumn{6}{|l|}{ Healthy lifestyle characteristics } \\
\hline Alcohol consumption (less than one drink per day) & 511 & $(83.0)$ & 599 & $(83.7)$ & \\
\hline Smoking behavior (non-smoker) & 529 & $(84.9)$ & 643 & $(88.8)$ & * \\
\hline Exercise frequency (twice or more per week) & 179 & $(28.8)$ & 261 & $(36.2)$ & ** \\
\hline Body mass index (18.5 - 24.9) & 403 & $(64.7)$ & 459 & $(63.3)$ & \\
\hline Sleep duration (6 - 9 hours) & 498 & $(80.1)$ & 610 & $(84.5)$ & * \\
\hline Breakfast (every morning) & 579 & $(93.2)$ & 682 & $(95.3)$ & \\
\hline Snack between meals (no) & 546 & $(87.9)$ & 636 & $(88.2)$ & \\
\hline Total number of healthy lifestyle items & 5.2 & $(1.1)$ & 5.4 & $(1.1)$ & \\
\hline Proportion of participants with 6 or 7 healthy lifestyle items & 268 & $(43.5)$ & 371 & $(52.3)$ & ** \\
\hline \multicolumn{6}{|l|}{ Health literacy } \\
\hline Seeking information from various sources & 3.0 & $(1.0)$ & 4.2 & $(0.5)$ & ** \\
\hline Extracting relevant information & 2.7 & $(0.9)$ & 4.1 & $(0.5)$ & ** \\
\hline Understanding and communicating the information & 2.6 & $(1.0)$ & 4.0 & $(0.6)$ & ** \\
\hline Considering the credibility of the information & 2.7 & $(0.9)$ & 3.9 & $(0.6)$ & ** \\
\hline Making decisions based on the information & 2.7 & $(0.9)$ & 3.9 & $(0.6)$ & ** \\
\hline Total score & 13.6 & $(3.2)$ & 20.0 & $(1.7)$ & ** \\
\hline
\end{tabular}

$\mathrm{a}^{* * *} \mathrm{P}<0.01,{ }^{*} \mathrm{P}<0.05$. 
66.4 years and $48.0 \%$ in the low HL group, and 66.5 years and $43.3 \%$ in the high HL group, respectively. HDL-C concentration in the high HL group was significantly higher than in the low HL group. Among healthy lifestyle characteristics, proportions of smoking behavior (non-smoker), exercise frequency (twice or more per week), and sleep duration (7 - 8 hours) were significantly higher in the high HL group than in the low HL group. In addition, the proportion of participants with $6-7$ total healthy lifestyle items was significantly higher in the high HL group.

Participants with seven healthy lifestyle characteristics had significantly higher HL compared to those with five or fewer healthy lifestyle items (Figure 1).

Table 2 shows age-specific multivariate regression analyses for non-elderly and elderly participants. The results show that high HL (total scale score $\geq 18$ ) was significantly associated with having $6-7$ healthy lifestyle characteristics in Model 1 among non-elderly (Odds ratio [OR] = 1.40, 95\% Confidence interval [CI] = 1.13 1.75) (Table 2(a)) and elderly (OR $=1.34,95 \% \mathrm{CI}=1.02-1.76)$ participants (Table 2(b)). In particular, Model 2 shows that scores of four or higher for HL items "Extracting relevant information" (OR $=1.52,95 \%$ CI $=1.11$ - 2.07) and "Making decisions based on the information" ( $\mathrm{OR}=1.45,95 \% \mathrm{CI}=1.11-1.89$ ) were significantly associated with healthy life characteristics among non-elderly participants (Table 2(a)). Among elderly participants, a score of at least four on "Extracting relevant information" $(\mathrm{OR}=1.55,95 \% \mathrm{CI}=1.04-2.30)$ was significantly associated with healthy lifestyle characteristics (Table 2(b)).

Table 3 shows sex-specific logistic regression analyses for male and female participants. High HL $(\geq 18)$ showed a significant association with 6 - 7 healthy lifestyle characteristics for female participants $(\mathrm{OR}=1.43$, $95 \% \mathrm{CI}=1.06$ - 1.94) $($ Table $3(\mathrm{~b}))$ and borderline significance for male participants $(\mathrm{OR}=1.34,95 \% \mathrm{CI}=0.96$ - 1.88) (Table 3(a)). Model 2 revealed that a score of four or higher on "Extracting relevant information"

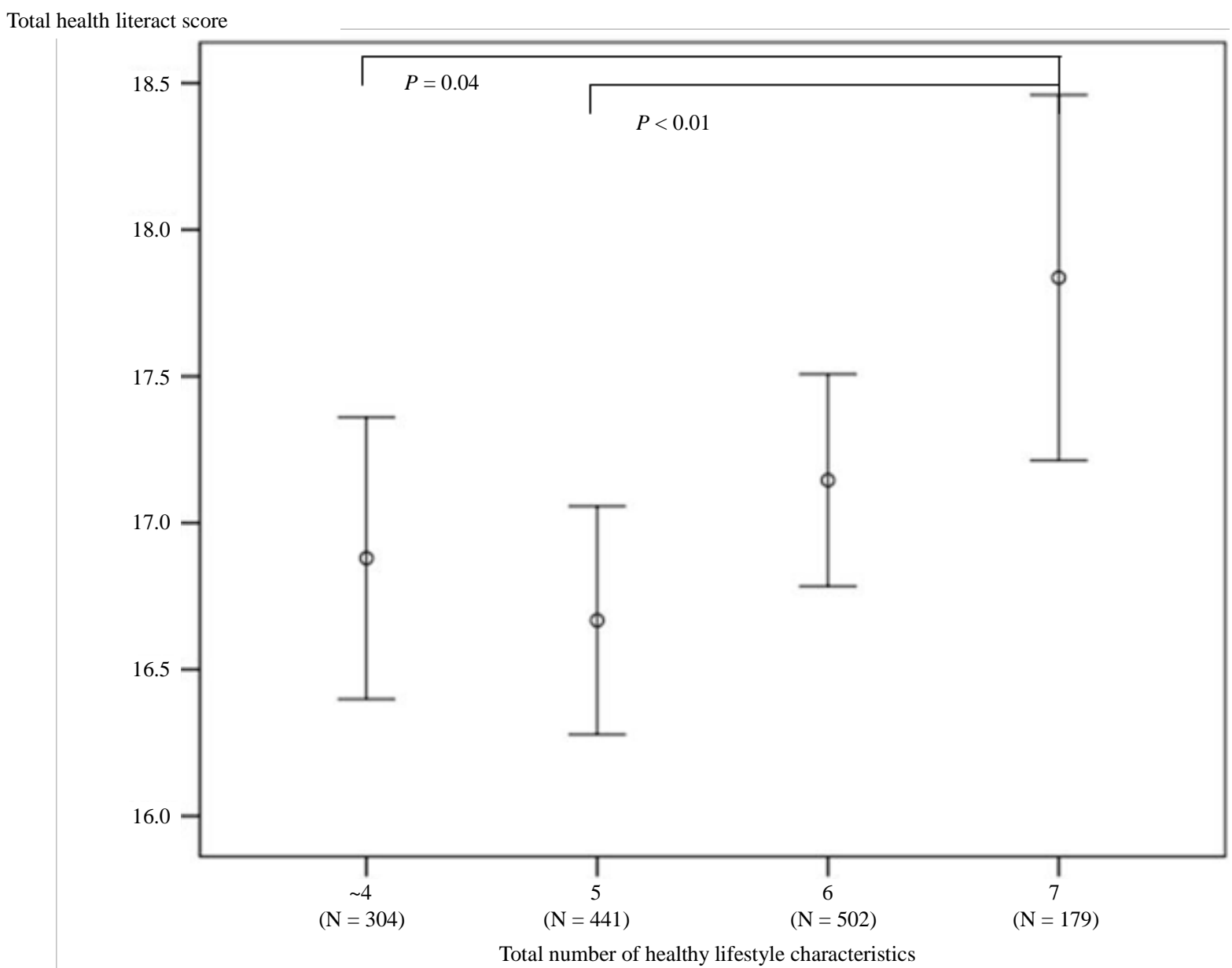

Figure 1. Correlation between total health literacy (HL) score and total number of healthy lifestyle characteristics. 
Table 2. (a) Logistic regression analysis of health literacy for non-elderly participants ( $<65$ years old) with $6-7$ healthy lifestyle characteristics $(\mathrm{N}=462)$; (b) Logistic regression analysis of health literacy for elderly participants ( $\geq 65$ years old) with 6 - 7 healthy lifestyle characteristics $(\mathrm{N}=886)$.

(a)

\begin{tabular}{|c|c|c|c|c|c|c|c|c|c|c|}
\hline & \multicolumn{4}{|c|}{ Univariate analysis } & \multicolumn{3}{|c|}{ Model $1^{\mathrm{c}}$} & \multicolumn{3}{|c|}{ Model $2^{\mathrm{d}}$} \\
\hline & N (\%) & $\mathrm{OR}^{\mathrm{a}}$ & $95 \% \mathrm{CI}^{\mathrm{b}}$ & $P^{\mathrm{e}}$ & $\mathrm{OR}^{\mathrm{a}}$ & $95 \% \mathrm{CI}^{\mathrm{b}}$ & $P^{\mathrm{e}}$ & $\mathrm{OR}^{\mathrm{a}}$ & $95 \% \mathrm{CI}^{\mathrm{b}}$ & $P^{\mathrm{e}}$ \\
\hline \multicolumn{11}{|l|}{ Health literacy } \\
\hline $\begin{array}{l}\text { Seeking information from various } \\
\text { sources ( } \geq 4 \text { vs. }<4 \text { ) }\end{array}$ & $721(81.6)$ & 1.15 & $0.91-1.45$ & & - & - & & 0.77 & $0.57-1.05$ & \\
\hline $\begin{array}{l}\text { Extracting relevant information } \\
(\geq 4 \text { vs. }<4)\end{array}$ & 802 (90.7) & 1.45 & $1.17-1.81$ & ** & - & - & & 1.52 & $1.11-2.07$ & ** \\
\hline $\begin{array}{l}\text { Understanding and communicating the } \\
\text { information }(\geq 4 \text { vs. }<4)\end{array}$ & $447(50.6)$ & 1.28 & $1.03-1.59$ & * & - & - & & 0.95 & $0.71-1.26$ & \\
\hline $\begin{array}{l}\text { Considering the credibility of the } \\
\text { information ( } \geq 4 \text { vs. }<4 \text { ) }\end{array}$ & $473(53.5)$ & 1.27 & $1.02-1.57$ & * & - & - & & 0.97 & $0.73-1.28$ & \\
\hline $\begin{array}{l}\text { Making decisions based on the } \\
\text { information ( } \geq 4 \text { vs. }<4 \text { ) }\end{array}$ & $729(82.5)$ & 1.59 & $1.28-1.97$ & ** & - & - & & 1.45 & $1.11-1.89$ & ** \\
\hline Total score $(\geq 18$ vs. $<18)$ & $867(98.1)$ & 1.43 & $1.15-1.77$ & ** & 1.40 & $1.13-1.75$ & ** & - & - & \\
\hline Sex (female vs. male) & $538(60.9)$ & - & - & & 1.78 & $1.43-2.23$ & ** & 1.79 & $1.42-2.24$ & ** \\
\hline Atherosclerotic complications & & - & - & & & & & & & \\
\hline Cardiovascular disease (present) & $4(0.9)$ & - & - & & 0.98 & $0.54-1.80$ & & 1.01 & $0.55-1.86$ & \\
\hline Cerebrovascular disease (present) & $2(0.4)$ & - & - & & 0.81 & $0.41-1.60$ & & 0.77 & $0.39-1.52$ & \\
\hline Medical history & & - & - & & & & & & & \\
\hline Antihypertensive drug use (yes) & $65(14.1)$ & - & - & & 0.99 & $0.79-1.26$ & & 1.01 & $0.80-1.28$ & \\
\hline Antidiabetic drug use (yes) & $7(1.5)$ & - & - & & 0.70 & $0.37-1.30$ & & 0.72 & $0.39-1.35$ & \\
\hline Antidyslipidemic drug use (yes) & $27(5.8)$ & - & - & & 1.17 & $0.85-1.61$ & & 1.17 & $0.85-1.61$ & \\
\hline
\end{tabular}

(b)

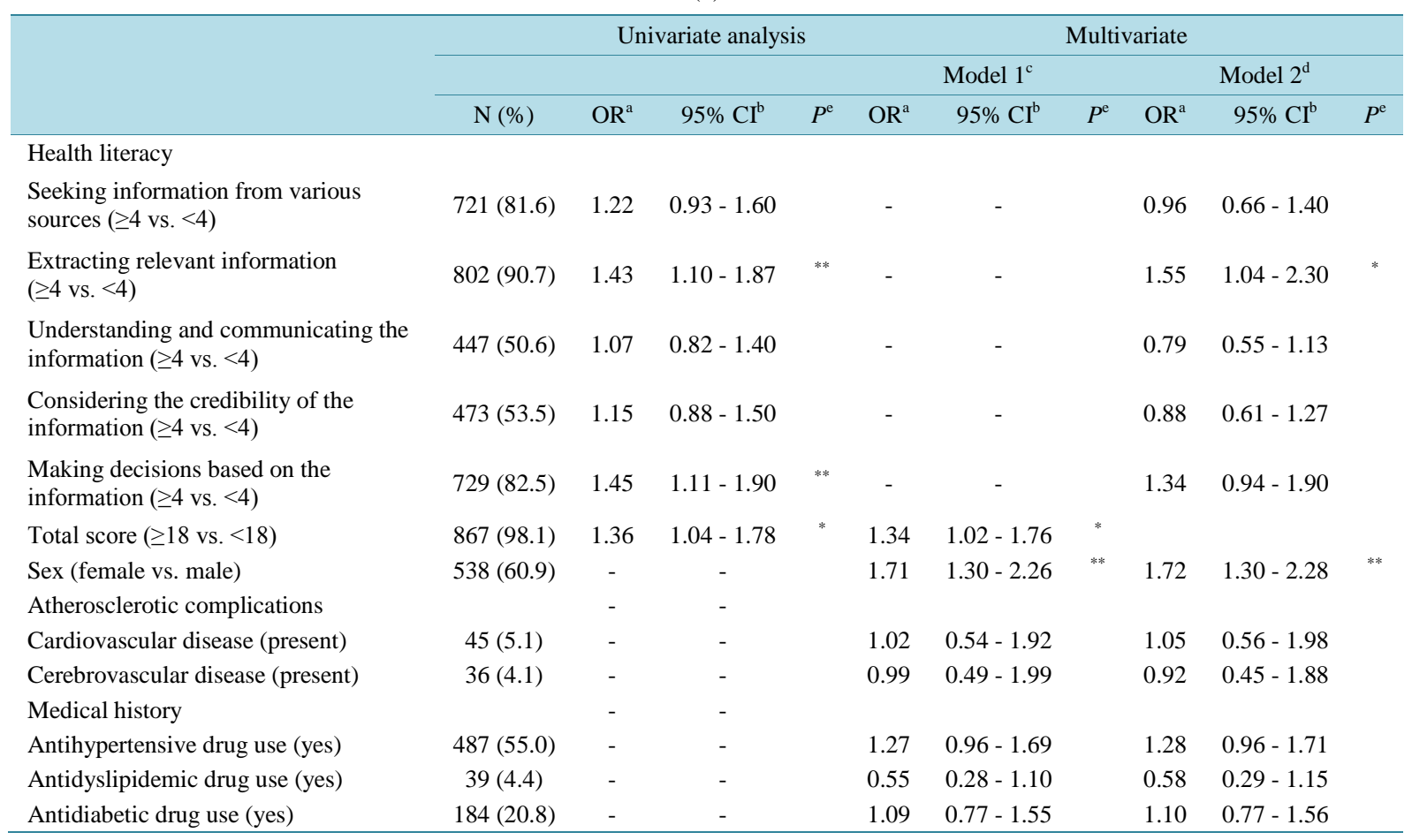

${ }^{a}$ Odds ratio, ${ }^{b} 95 \%$ confidence interval, ${ }^{c}$ Model 1 was adjusted for sex, total health literacy score $(\geq 18$ vs. $<18$ ), atherosclerotic complications (cardiovascular and cerebrovascular diseases), and medical history of lifestyle-related disorders (antihypertensive drug use, antidiabetic drug use, antidyslipidemic drug use); ${ }^{\mathrm{d}}$ Model 2 was adjusted for sex, 5 health literacy items ( $\geq 4$ vs. $<4$ )), atherosclerotic complications (cardiovascular and cerebrovascular diseases), and medical history of lifestyle-related disorders (antihypertensive drug use, antidiabetic drug use, antidyslipidemic drug use); ${ }^{* * *} P<$ $0.01,{ }^{*} P<0.05$ 
Table 3. (a) Logistic regression analysis of health literacy for male participants with 6 - 7 healthy lifestyle characteristics (N = 613); (b) Logistic regression analysis of health literacy for female participants with $6-7$ healthy lifestyle characteristics ( $\mathrm{N}$ =735).

(a)

\begin{tabular}{|c|c|c|c|c|c|c|c|c|c|c|}
\hline & \multicolumn{6}{|c|}{ Univariate analysis } & \multicolumn{4}{|c|}{ Multivariate } \\
\hline & \multirow[b]{2}{*}{ N (\%) } & \multirow[b]{2}{*}{$\mathrm{OR}^{\mathrm{a}}$} & \multirow[b]{2}{*}{$95 \% \mathrm{CI}^{\mathrm{b}}$} & \multirow[b]{2}{*}{$P^{\mathrm{e}}$} & \multicolumn{3}{|c|}{ Model $1^{\mathrm{c}}$} & \multicolumn{3}{|c|}{ Model $2^{d}$} \\
\hline & & & & & $\mathrm{OR}^{\mathrm{a}}$ & $95 \% \mathrm{CI}^{\mathrm{b}}$ & $P^{\mathrm{e}}$ & $\mathrm{OR}^{\mathrm{a}}$ & $95 \% \mathrm{CI}^{\mathrm{b}}$ & $P^{\mathrm{e}}$ \\
\hline \multicolumn{11}{|l|}{ Health literacy } \\
\hline $\begin{array}{l}\text { Seeking information from various } \\
\text { sources }(\geq 4 \text { vs. }<4)\end{array}$ & $402(65.6)$ & 1.19 & $0.85-1.67$ & & - & - & & 1.09 & $0.68-1.76$ & \\
\hline $\begin{array}{l}\text { Extracting relevant information } \\
(\geq 4 \text { vs. }<4)\end{array}$ & $353(57.6)$ & 1.39 & $1.00-1.93$ & * & - & - & & 1.42 & $0.86-2.34$ & \\
\hline $\begin{array}{l}\text { Understanding and communicating the } \\
\text { information }(\geq 4 \text { vs. }<4)\end{array}$ & $313(51.1)$ & 1.24 & $0.89-1.71$ & & - & - & & 0.85 & $0.54-1.34$ & \\
\hline $\begin{array}{l}\text { Considering the credibility of the } \\
\text { information }(\geq 4 \text { vs. }<4)\end{array}$ & 306 (49.9) & 1.31 & $0.95-1.81$ & & - & - & & 0.92 & $0.58-1.44$ & \\
\hline $\begin{array}{l}\text { Making decisions based on the } \\
\text { information }(\geq 4 \text { vs. }<4) \text { ) }\end{array}$ & $282(46.0)$ & 1.54 & $1.11-2.13$ & ** & - & - & & 1.26 & $0.83-1.90$ & \\
\hline Total score $(\geq 18$ vs. $<18)$ & $314(51.2)$ & 1.42 & $1.02-2.00$ & * & 1.34 & $0.96-1.88$ & 0.09 & & - & \\
\hline Age (years old) & & - & - & & 1.04 & $1.02-1.05$ & ${ }^{* *}$ & 1.04 & $1.02-1.05$ & ** \\
\hline \multicolumn{11}{|l|}{ Atherosclerotic complications } \\
\hline Cardiovascular disease (present) & $30(4.9)$ & - & - & & 0.78 & $0.35-1.76$ & & 0.78 & $0.35-1.75$ & \\
\hline Cerebrovascular disease (present) & $26(4.2)$ & - & - & & 0.58 & $0.24-1.42$ & & 0.62 & $0.25-1.53$ & \\
\hline Medical history & & - & - & & & & & & & \\
\hline Antihypertensive drug use (yes) & 242 (39.5) & - & - & & 0.72 & $0.49-1.07$ & & 0.72 & $0.49-1.07$ & \\
\hline Antidyslipidemic drug use (yes) & $74(12.1)$ & - & - & & 1.18 & $0.68-2.04$ & & 1.18 & $0.68-2.05$ & \\
\hline Antidiabetic drug use (yes) & $31(5.1)$ & - & - & & 0.73 & $0.34-1.57$ & & 0.73 & $0.34-1.58$ & \\
\hline
\end{tabular}

(b)

\begin{tabular}{|c|c|c|c|c|c|c|c|c|c|c|}
\hline & \multicolumn{6}{|c|}{ Univariate analysis } & \multicolumn{4}{|c|}{ Multivariate } \\
\hline & \multirow[b]{2}{*}{ N (\%) } & \multirow[b]{2}{*}{$\mathrm{OR}^{\mathrm{a}}$} & \multirow[b]{2}{*}{$95 \% \mathrm{CI}^{\mathrm{b}}$} & \multirow[b]{2}{*}{$P^{\mathrm{e}}$} & \multicolumn{3}{|c|}{ Model $1^{\mathrm{c}}$} & \multicolumn{3}{|c|}{ Model $2^{\mathrm{d}}$} \\
\hline & & & & & $\mathrm{OR}^{\mathrm{a}}$ & $95 \% \mathrm{CI}^{\mathrm{b}}$ & $P^{\mathrm{e}}$ & $\mathrm{OR}^{\mathrm{a}}$ & $95 \% \mathrm{CI}^{\mathrm{b}}$ & $P^{\mathrm{e}}$ \\
\hline \multicolumn{11}{|l|}{ Health literacy } \\
\hline $\begin{array}{l}\text { Seeking information from various } \\
\text { sources }(\geq 4 \text { vs. }<4)\end{array}$ & $519(70.6)$ & 1.02 & $0.74-1.40$ & & - & - & & 0.81 & $0.53-1.23$ & \\
\hline $\begin{array}{l}\text { Extracting relevant information } \\
(\geq 4 \text { vs. }<4)\end{array}$ & $423(57.6)$ & 1.53 & $1.14-2.05$ & ** & - & - & & 1.95 & $1.28-2.97$ & ** \\
\hline $\begin{array}{l}\text { Understanding and communicating the } \\
\text { information }(\geq 4 \text { vs. }<4)\end{array}$ & $424(57.7)$ & 1.21 & $0.90-1.62$ & & - & - & & 0.91 & $0.62-1.35$ & \\
\hline $\begin{array}{l}\text { Considering the credibility of the } \\
\text { information ( } \geq 4 \text { vs. }<4 \text { ) }\end{array}$ & 388 (52.8) & 1.21 & $0.91-1.62$ & & - & - & & 0.83 & $0.56-1.22$ & \\
\hline $\begin{array}{l}\text { Making decisions based on the } \\
\text { information ( } \geq 4 \text { vs. }<4)\end{array}$ & 417 (56.7) & 1.52 & $1.13-2.04$ & ** & - & - & & 1.30 & $0.90-1.88$ & \\
\hline Total score $(\geq 18$ vs. $<18)$ & 411 (55.9) & 1.38 & $1.03-1.86$ & $*$ & 1.43 & $1.06-1.94$ & * & & - & \\
\hline Age (years old) & & - & - & & 1.03 & $1.01-1.04$ & ** & 2.34 & $1.61-3.40$ & ** \\
\hline \multicolumn{11}{|l|}{ Atherosclerotic complications } \\
\hline Cardiovascular disease (present) & $19(2.6)$ & - & - & & 0.76 & $0.29-2.02$ & & 0.47 & $0.26-1.86$ & \\
\hline Cerebrovascular disease (present) & $12(1.6)$ & - & - & & 4.29 & $0.91-20.3$ & & 4.15 & $0.87-19.91$ & \\
\hline \multicolumn{11}{|l|}{ Medical history } \\
\hline Antihypertensive drug use (yes) & $310(42.2)$ & - & - & & 0.66 & $0.47-0.94$ & * & 0.68 & $0.48-0.97$ & \\
\hline Antidyslipidemic drug use (yes) & $137(18.6)$ & - & - & & 1.02 & $0.68-1.53$ & & 0.98 & $0.65-1.47$ & \\
\hline Antidiabetic drug use (yes) & $15(2.0)$ & - & - & & 0.59 & $0.20-1.73$ & & 0.64 & $0.22-1.91$ & \\
\hline
\end{tabular}

${ }^{\mathrm{a} O d d s}$ ratio, ${ }^{\mathrm{b}} 95 \%$ confidence interval, ${ }^{\mathrm{c}}$ Model 1 was adjusted for age (years old), total health literacy score ( $\geq 18$ vs. $<18$ ), atherosclerotic complications (cardiovascular and cerebrovascular diseases), and medical history of lifestyle-related disorders (antihypertensive drug use, antidiabetic drug use, antidyslipidemic drug use); ${ }^{\mathrm{d}}$ Model 2 was adjusted for age (years old), 5 health literacy items ( $\geq 4$ vs. $<4$ ), atherosclerotic complications (cardiovascular and cerebrovascular diseases), and medical history of lifestyle-related disorders (antihypertensive drug use, antidiabetic drug use, antidyslipidemic drug use); ${ }^{* *} P<0.01,{ }^{*} P<0.05$. 
was significantly associated $(\mathrm{OR}=1.95,95 \% \mathrm{CI}=1.28$ - 2.97) with healthy lifestyle characteristics among female participants (Table 3(b)).

\section{Discussion}

Analysis of cross-sectional data showed that high HL was significantly associated with healthy lifestyle characteristics, specifically non-smoker status, high exercise frequency, and adequate sleep (Table 1). Multivariate analyses revealed that high HL $(\geq 18)$ was significantly associated with having 6 - 7 healthy lifestyle characteristics in both age categories and female participants, while the significance of the association was a borderline in male participants. In addition, the ability to extract health-related information was significantly associated with a healthy lifestyle regardless of age or sex. Although several studies have examined HL characteristics of young and middle-aged participants [9], male participants [9], and patients with diabetes mellitus [16], there have been a few reports that broadly include elderly and female participants. To the best of our knowledge, the present study is the first to describe age- and sex-specific HL characteristics among Japanese community-dwelling residents, and to examine associations with healthy lifestyle characteristics.

Only a few studies have examined the association between HL and health behavior [9] [17]. These reports have indicated that HL is one of the potential intermediate factors linked with health-related behavior. A study of 2,824 Australians aged 15 years or older reported that inadequate functional HL is associated with 2 - 3 (OR $=1.9)$ and 4 or more $(\mathrm{OR}=2.8)$ self-reported lifestyle risk factors [17]. A pilot study that evaluated a newly-developed, brief measurement of HL among Japanese office workers showed that people with higher HL are more likely to have regular eating patterns and exercise weekly, and to never have smoked [9]. Consistent with these reports, our results showed that participants in the high HL group were likely to be non-smokers, exercise frequently, and get adequate sleep. Furthermore, multivariate analyses showed significant associations between healthy lifestyle characteristics and high HL. Thus, the previous studies and our results indicate an association between $\mathrm{HL}$ and lifestyle characteristics, and emphasize the importance of improving HL for maintaining a healthy lifestyle.

We also conducted analyses among the Japanese community-dwelling population considering with age and sex. A few reports for the elderly can be found worldwide [18], and previous Japanese studies were designed for non-elderly participants or office workers in which the proportion of females was relatively small. Populations are rapidly aging worldwide, and this is particularly true in Japan where there is an increased need to address problems associated with the aging population [19]. Thus, the elderly population must be considered for adequate health promotion in the community. Our results highlight the association between HL and lifestyle characteristics among elderly and non-elderly participants. HL was also positively associated with healthy lifestyle characteristics among female participants and the association was not observed among male participants. In the present study, female participants were likely to have better HL and healthy characteristics compared to male participants (mean of HL; 16.8 for male and 17.3 for female, mean of total healthy lifestyle characteristics; 5.1 for male and 5.5 for female). The difference among both sexes may be related with levels of HL and healthy lifestyle characteristics at baseline.

This study has several limitations. First, there may have been selection bias, as study participants were recruited from those who participated in a multi-phasic health checkup program at a single Japanese village. These participants may have had higher health awareness compared to non-participants. Large scale studies that include several regions including more non-participants will be needed in the future. Second, HL and lifestyle characteristics were measured based on self-reported questionnaires. It is possible that these participants may report better own HL and healthy lifestyles than these are. This may have resulted in over-estimation of HL and health characteristics. Third, given the cross-sectional design, we could not determine whether there was a causal relationship between HL and healthy lifestyle characteristics. A longitudinal study will be needed to address this issue. Fourth, while the information collected from participants contained a comprehensive set of clinical variables, it cannot be denied that some important factors, especially educational status, were not measured. The inclusion of additional factors in the multivariate analysis might have produced different results.

\section{Conclusion}

The present study revealed a positive association between HL and healthy lifestyle characteristics among community-dwelling participants, which suggests that people are likely to engage in health-promoting behaviors 
based on access and ability to understand medical information. Our results underscore the importance of comprehensive assessment, including HL, for health promotion in the community.

\section{Acknowledgements}

We thank the staff at Tsumagoi Village for their excellent help in data collection. This study was funded by a 2012 Grant-in-Aid for Scientific Research (C) (No. 24590816).

\section{References}

[1] Lozano, R., Naghavi, M., Foreman K., et al. (2012) Global and Regional Mortality from 235 Causes of Death for 20 Age Groups in 1990 and 2010: A Systematic Analysis for the Global Burden of Disease Study 2010. Lancet, 380, 2095-2128. http://dx.doi.org/10.1016/S0140-6736(12)61728-0

[2] Umpierre, D., Ribeiro, P.A., Kramer, C.K., Leitão, C.B., Zucatti, A.T., Azevedo, M.J., Gross, J.L., Ribeiro, J.P. and Schaan, B.D. (2011) Physical Activity Advice Only or Structured Exercise Training and Association with HbA1c Levels in Type 2 Diabetes: A Systematic Review and Meta-Analysis. JAMA, 30, 1790-1799. http://dx.doi.org/10.1001/jama.2011.576

[3] Rutten, L.J., Arora, N.K., Bakos, A.D., Aziz, N. and Rowland, J. (2005) Information Needs and Sources of Information among Cancer Patients: A Systematic Review of Research (1980-2003). Patient Education and Counseling, 57, 250261. http://dx.doi.org/10.1016/j.pec.2004.06.006

[4] Hesse, B.W., Nelson, D.E., Kreps, G.L., Croyle, R.T., Arora, N.K., Rimer, B.K. and Viswanath, K. (2005) Trust and Sources of Health Information: The Impact of the Internet and Its Implications for Health Care Providers: Findings from the First Health Information National Trends Survey. Archives of Internal Medicine, 165, 2618-2624. http://dx.doi.org/10.1001/archinte.165.22.2618

[5] Koch-Weser, S., Bradshaw, Y.S., Gualtieri, L. and Gallagher, S.S. (2010) The Internet as a Health Information Source: Findings from the 2007 Health Information National Trends Survey and Implications for Health Communication. Journal of Health Communication, 15, 279-293. http://dx.doi.org/10.1080/10810730.2010.522700

[6] Powers, B.J., Trinh. J.V. and Bosworth, H.B. (2010) Can This Patient Read and Understand Written Health Information? JAMA, 304, 76-84. http://dx.doi.org/10.1001/jama.2010.896

[7] Nutbeam, D. (2000) Health Literacy as a Public Health Goal: A Challenge for Contemporary Health Education and Communication Strategies into the 21st Century. Health Promotion International, 15, 259-267. http://dx.doi.org/10.1093/heapro/15.3.259

[8] Shibuya, A., Inoue, R., Ohkubo, T., Takeda, Y., Teshima, T., Imai, Y. and Kondo, Y. (2011) The Relation between Health Literacy, Hypertension Knowledge, and Blood Pressure among Middle-Aged Japanese Adults. Blood Pressure Monitoring, 16, 224-230. http://dx.doi.org/10.1097/MBP.0b013e32834af7ba

[9] Ishikawa, H., Nomura, K., Sato, M. and Yano, E. (2008) Developing a Measure of Communicative and Critical Health Literacy: A Pilot Study of Japanese Office Workers. Health Promotion International, 23, 269-274. http://dx.doi.org/10.1093/heapro/dan017

[10] Suka, M., Odajima, T., Kasai, M., Igarashi, A., Ishikawa, H., Kusama, M., Nakayama, T., Sumitani, M. and Sugimori, H. (2013) The 14-Item Health Literacy Scale for Japanese Adults (HLS-14). Environmental Health and Preventive Medicine, 18, 407-415. http://dx.doi.org/10.1007/s12199-013-0340-z

[11] Warnick, G.R., Knopp, R.H., Fitzpatrick, V. and Branson, L. (1990) Estimating Low-Density Lipoprotein Cholesterol by the Friedewald Equation Is Adequate for Classifying Patients on the Basis of Nationally Recommended Cut Points. Clinical Chemistry, 36, 15-19.

[12] Kashiwagi, A., Kasuga, M., Araki, E., Oka, Y., Hanafusa, T., Ito, H., Tominaga, M., Oikawa, S., Noda, M., Kawamura, T., Sanke, T., Namba, M., Hashiramoto, M., Sasahara, T., Nishio, Y., Kuwa, K., Ueki, K., Takei, I., Umemoto, M., Murakami, M., Yamakado, M., Yatomi, Y. and Ohashi, H., Committee on the Standardization of Diabetes MellitusRelated Laboratory Testing of Japan Diabetes Society (2012) International Clinical Harmonization of Glycated Hemoglobin in Japan: From Japan Diabetes Society to National Glycohemoglobin Standardization Program Values. Journal of Diabetes Investigation, 3, 39-40. http://dx.doi.org/10.1111/j.2040-1124.2012.00207.x

[13] Belloc, N.B. and Breslow, L. (1972) Relationship of Physical Health Status and Health Practices. Preventive Medicine, 1, 409-421. http://dx.doi.org/10.1016/0091-7435(72)90014-X

[14] Yokokawa, H., Goto, A., Sanada, H., Watanabe, T., Felder, R.A., Jose, P.A. and Yasumura S. (2011) Achievement Status toward Goal Blood Pressure Levels and Healthy Lifestyles among Japanese Hypertensive Patients; Cross-Sectional Survey Results from Fukushima Research of Hypertension (FRESH). Internal Medicine, 50, 1149-1156. http://dx.doi.org/10.2169/internalmedicine.50.4969 
[15] Ministry of Health, Labor and Welfare \& Ministry of Education, Culture, Sports, Science and Technology. Ethical Guideline for Epidemiological Studies (2013). (In Japanese) http://www.mhlw.go.jp/general/seido/kousei/i-kenkyu/ekigaku/0504sisin.html

[16] Ishikawa, H., Takeuchi, T. and Yano, E. (2008) Measuring Functional, Communicative, and Critical Health Literacy among Diabetic Patients. Diabetes Care, 31, 874-879. http://dx.doi.org/10.2337/dc07-1932

[17] Adams, R.J., Piantadosi, C., Ettridge, K., Miller, C., Wilson, C., Tucker, G. and Hill, C.L. (2013) Functional Health Literacy Mediates the Relationship between Socio-Economic Status, Perceptions and Lifestyle Behaviors Related to Cancer Risk in an Australian Population. Patient Education and Counseling, 91, 206-212. http://dx.doi.org/10.1016/j.pec.2012.12.001

[18] Mullen, E. (2013) Health Literacy Challenges in the Aging Population. Nursing Forum, 48, 248-255. http://dx.doi.org/10.1111/nuf.12038

[19] (2014) United Nations, Department of Economic and Social Affairs, Population Division. World Population Prospects; The 2012 Revision 2014. http://esa.un.org/unpd/wpp/Documentation/pdf/WPP2012 HIGHLIGHTS.pdf 